\title{
Obesity, hypertension and aldosterone: is leptin the link?
}

\author{
Ding Xie ${ }^{1}$ and Wendy B Bollag ${ }^{2,3}$ \\ 1Department of Family Medicine, Medical College of Georgia at Augusta University (formerly Georgia \\ Regents University), Augusta, Georgia, USA \\ ${ }^{2}$ Charlie Norwood VA Medical Center, Augusta, Georgia, USA \\ ${ }^{3}$ Department of Physiology, Medical College of Georgia at Augusta University (formerly Georgia \\ Regents University), Augusta, Georgia, USA
}

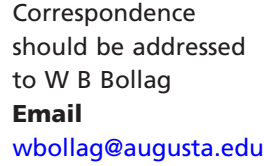

\begin{abstract}
Obesity is a serious health hazard with rapidly increasing prevalence in the United States. In 2014, the World Health Organization estimated that nearly 2 billion people worldwide were overweight with an estimated 600 million of these obese. Obesity is associated with many chronic diseases, including cardiovascular disease and hypertension. Data from the Framingham Heart study suggest that approximately $78 \%$ of the risk for hypertension in men and $65 \%$ in women is related to excess body weight, a relationship that is further supported by studies showing increases in blood pressure with weight gain and decreases with weight loss. However, the exact mechanism by which excess body fat induces hypertension remains poorly understood. Several clinical studies have demonstrated elevated plasma aldosterone levels in obese individuals, especially those with visceral adiposity, with decreased aldosterone levels measured in concert with reduced blood pressure following weight loss. Since aldosterone is a mineralocorticoid hormone that regulates blood volume and pressure, serum aldosterone levels may link obesity and hypertension. Nevertheless, the mechanism by which obesity induces aldosterone production is unclear. A recent study by Belin de Chantemele and coworkers suggests that one adipose-released factor, leptin, is a direct agonist for aldosterone secretion; other adipose-related factors may also contribute to elevated aldosterone levels in obesity, such as very low-density lipoprotein (VLDL), the levels of which are elevated in obesity and which also directly stimulates aldosterone biosynthesis. This focused review explores the possible roles of leptin and VLDL in modulating aldosterone secretion to underlie obesity-associated hypertension.
\end{abstract}

It is well known that obesity is associated with a number of metabolic end organ dysfunctions, including hypertension. However, less well understood is the mechanism by which obesity leads to elevated blood pressure. Various hypotheses have been proposed including increased sympathetic nervous system activity, overactivation of the renin-angiotensin II-aldosterone system, excessive secretion of adipose-derived cytokines, inflammation and compression of the kidneys by excess perirenal fat deposits (Canale et al. 2013). On the other hand, accumulating evidence suggests a potential link between obesity-related hypertension and the 


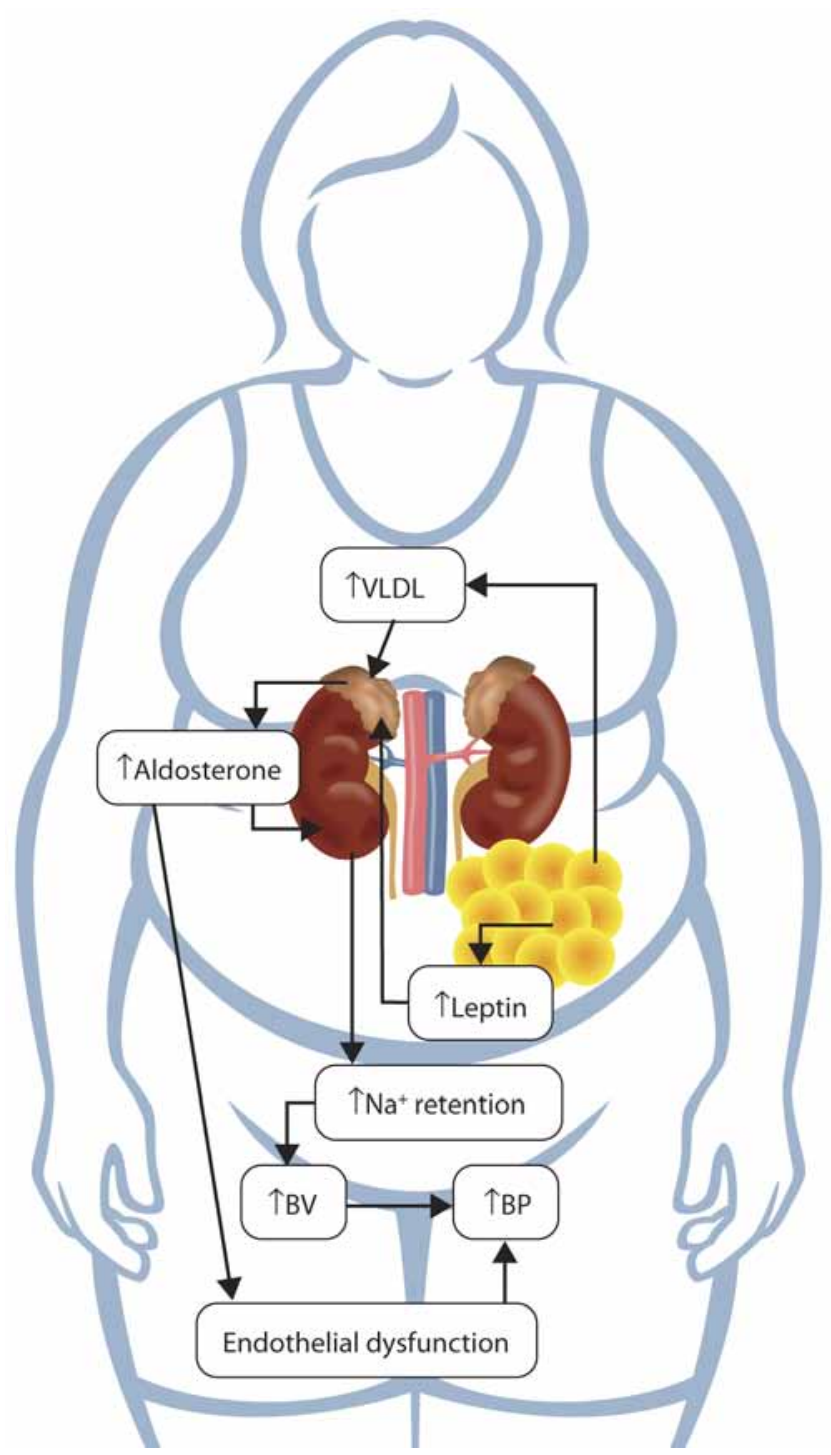

Figure 1

Obesity increases blood pressure. Excess fat deposits (including those surrounding the adrenal glands) of obese individuals secrete large amounts of leptin. The data of Belin de Chantemele and coworkers indicate that this adipose-derived leptin can stimulate zona glomerulosa cells of the adrenal gland to produce aldosterone. Aldosterone, in turn, causes the kidney to retain salt and water, raising blood volume and pressure. Aldosterone can also produce endothelial dysfunction and cardiac fibrosis. Obesity also results in dyslipidemia, including increased serum levels of VLDL; this lipoprotein has also been shown to induce aldosterone production. Other mechanisms (not illustrated) that potentially underlie obesity-associated hypertension include increased levels of proinflammatory cytokines, hypersecretion of various adipokines and/or excessive activity of the sympathetic nervous or renin-angiotensin II-aldosterone systems.

mineralocorticoid aldosterone (Briet \& Schiffrin 2011), a steroid hormone involved in maintaining fluid and electrolyte balance in the human body. Thus, aldosterone levels are elevated in obesity, and weight loss in obese individuals is accompanied by reductions in both blood pressure and aldosterone levels (Calhoun \& Sharma 2010, Briet \& Schiffrin 2011). Furthermore, in several studies, aldosterone levels have been correlated with body mass index, waist circumference and/or visceral adiposity (Calhoun \& Sharma 2010). Based on this link between obesity and aldosterone levels, it has been proposed that adipose tissue produces a factor or factors that can stimulate aldosterone secretion from the adrenal gland independently of the traditional aldosterone secretagogues, angiotensin II (AngII), elevated serum potassium levels and adrenocorticotrophic hormone (ACTH). Nevertheless, the identity of this adipose-secreted factor is unclear.

Since the discovery of leptin as an adipocyte-derived satiety factor, adipose tissue is no longer viewed simply as a passive energy storage site but also as an active endocrine and paracrine organ secreting an ever-increasing number of mediators, termed adipokines. Research indicates that adipocytes communicate with other organs through these adipokines and participate in diverse metabolic processes including cardiovascular function (Rondinone 2006). Leptin, a protein hormone secreted predominantly by adipocytes in proportion to fat mass, plays a role in the regulation of food intake and a series of other physiologic actions, including blood pressure control, through its widely distributed receptors (Rondinone 2006). It has been previously demonstrated that leptin receptors are expressed in human and rat adrenal glomerulosa cells (Ehrhart-Bornstein et al. 2003), although available data on the direct effects of leptin on aldosterone secretion in vitro are controversial (e.g., Malendowicz et al. 1997 vs Salzmann et al. 2004). Nevertheless, obesity is characterized by concomitant hyperleptinemia and hyperaldosteronism, and decreases in both aldosterone and leptin are observed with weight reduction, suggesting a possible interaction of these two hormones (Engeli \& Sharma 2000). Indeed, a recent article from the laboratory of Belin de Chantemele (Huby et al. 2015) provides strong evidence, both in vitro and in vivo, that the adipokine leptin is an important aldosterone stimulatory factor released from adipose tissue (Fig. 1).

In this article, Huby and coworkers (2015) first demonstrated that leptin receptors can be localized to aldosterone synthase (CYP11B2)-positive zona glomerulosa cells in human adrenal glands, and are present in isolated rat adrenocortical cells and the human adrenocortical carcinoma cell line, HAC15 cells. Leptin 
also dose-dependently increases aldosterone production and CYP11B2 protein levels in HAC15 adrenocortical carcinoma cells and rat adrenocortical cells in vitro in a leptin receptor-dependent manner. Leptin induces an increased cytosolic calcium concentration that is required for the observed changes in aldosterone and CYP11B2 levels. In addition, infusion of leptin in wildtype or ob/ob female mice lacking leptin in vivo results in a dose-dependent increase in serum aldosterone levels and aldosterone synthase protein expression without affecting serum sodium, potassium, AngII or ACTH levels. These elevations are inhibited by a leptin receptor antagonist, but not AngII type 1 (AT1R) or adrenergic receptor antagonists, and leptin infusion into $\mathrm{db} / \mathrm{db}$ mice with nonfunctional leptin receptors does not produce such effects. Interestingly, while the ability of leptin to inhibit food intake becomes desensitized with continued hormone infusion, the effect of leptin to stimulate aldosterone secretion does not. Additional mouse models in which leptin or leptin signaling is enhanced also exhibit increased aldosterone and CYP11B2 protein levels: thus, these parameters are elevated in protein tyrosine phosphatase knockout mice, in which inactivation of leptin signaling is inhibited, in obese agouti mice with enhanced leptin levels and in high-fat-diet-induced obese mice, which also show higher leptin levels. Finally, leptin infusion into mice in vivo results in endothelial dysfunction and cardiac fibrosis, both of which can be reversed with the mineralocorticoid receptor (MR) antagonist spironolactone, suggesting that certain adverse effects of elevated leptin levels accompanying obesity may be attributable to this hormone's ability to increase aldosterone levels.

This study, which utilizes multiple in vivo rodent models as well as in vitro adrenocortical cell systems, strongly supports the idea that leptin is an adipose-derived factor that can directly promote aldosterone secretion. However, the experiments reported in this article were performed in female rodents; this group subsequently demonstrated that the aldosterone stimulatory effect of leptin underlies obesity-associated hypertension only in female mice, but not in males, in which leptin seems to exert its hypertensive effect instead through activation of the sympathetic nervous system (Huby et al. 2016). These data also seem to be consistent with results in humans, in that, for example, women exhibit a greater response to MR antagonists than men (Goodfriend et al. 1999). Nevertheless, despite the demonstrated, but possibly gender-specific, role of leptin in elevating aldosterone levels, it seems likely that leptin is not the only adipokine that regulates aldosterone production in the zona glomerulosa to contribute to obesity-associated hypertension.

Other cytokines released by adipose tissue, such as tumor necrosis factor- $\alpha$, interleukin-6, complement-C1q TNF-related protein 1 (CTRP1) and fatty acid oxidation products (Briet \& Schiffrin 2011), as well as additional factors known to be augmented in obesity, may also lead to elevated blood pressure through stimulated aldosterone secretion. For example, obesity is often associated with dyslipidemia, characterized by increased circulating plasma triglycerides, very low-density lipoprotein (VLDL) and low-density lipoprotein (LDL) levels (Briet \& Schiffrin 2011). This atherogenic dyslipidemia is an important contributor to the deleterious effects of being overweight or obese. VLDL has high triglyceride content (about 50\%) and serves to transport this lipid in the body; however, previous studies have shown that in addition to transporting lipids, VLDL can regulate signaling pathways in different tissue types. For example, VLDL modulates the expression in human endothelial cells of a variety of genes involved in, for example, cell signaling, cell transport and extracellular matrix formation and degradation (Norata et al. 2003). Oxidized VLDL regulates the expression of some of the same genes, but VLDL and oxidized VLDL each also alter the expression of their own set of genes, through activation of primarily extracellular signal-regulated kinase-1 and -2 (ERK1/2) in the case of VLDL and predominantly p38 mitogen-activated kinase with oxidized VLDL (Norata et al. 2003). These results, and others, indicate that VLDL can activate cell signaling pathways in various cell types in addition to transporting lipids throughout the body.

In recent studies, we showed in primary cultures of human adrenocortical and bovine glomerulosa cells, as well as the adrenocortical cell line, H295R, and its clone the HAC15 cell line, that VLDL significantly increases aldosterone synthesis in these various models of zona glomerulosa cells (Xing et al. 2012, Tsai et al. 2014). This stimulation occurs at physiological VLDL levels of $30-100 \mu \mathrm{g}$ protein $/ \mathrm{mL}$ and is largely due to VLDL's stimulation of the expression of steroidogenic acute regulatory protein and CYP11B2 (Xing et al. 2012). In the H295R cell line, the effects of VLDL on CYP11B2 transcript levels are not additive with high AngII or elevated extracellular potassium levels, but are additive with the cAMP-elevating agents АCTH and forskolin (Xing et al. 2012) and are not altered by exposure to an AT1R antagonist (Tsai et al. 2014). The effect of VLDL on aldosterone and CYP11B2 involves the calcium signaling pathway, as the changes can be inhibited by antagonists

Published by Bioscientifica Ltd. 
of voltage-dependent L-type calcium channels and calcium/calmodulin-dependent protein kinases (Xing et al. 2012). Phospholipase D has also been found to mediate aldosterone production and CYP11B2 expression in response to VLDL (Tsai et al. 2014), similarly to its role in AngII-induced steroidogenesis. Finally, we also showed that rats on a chow and liquid-sucrose diet that induces obesity and insulin resistance exhibit increased aldosterone synthase levels correlating with elevated triglyceride levels (a measure of VLDL levels in fasted animals) in vivo (Xing et al. 2012).

These results are similar to the data of Saha and coworkers, who showed that VLDL and oxidized VLDL stimulate aldosterone production. In vitro glycoxidized VLDL, which increases in diabetes (Saha et al. 2012b), also induces steroidogenesis (Saha et al. 2012a,b); VLDL prepared from individuals with impaired glucose tolerance stimulates aldosterone secretion as well (Saha et al. 2013). Using pharmacologic inhibitors, these authors also demonstrated that the effects of modified and unmodified VLDL are mediated by cAMP-dependent protein kinase, ERK1/2 and Janus kinase-2 through scavenger receptor class B, member 1 (Saha et al. 2012a). High-density lipoprotein also increases aldosterone production (Xing et al. 2011, Saha et al. 2012b), although its effects are less robust and more variable (Xing et al. 2012, Saha et al. $2012 b$ ). On the other hand, while some investigators have detected a steroidogenic effect of LDL (Saha et al. $2012 b$ ), the ability of this lipoprotein to stimulate aldosterone production remains controversial (Capponi 2002). Nevertheless, these data suggest the possibility that obesity-altered lipoproteins can also promote aldosterone production to contribute to hypertension.

Obesity-induced hypertension should be considered the most common form of essential hypertension. Indeed, data from the Framingham Heart Study suggest that about $78 \%$ of the risk for hypertension in men and approximately $65 \%$ in women can be directly related to excess body weight (Calhoun \& Sharma 2010). This relationship has been further supported by experimental studies showing an increase in blood pressure after weight gain, as well as the therapeutic value of weight loss in reducing blood pressure (Kawarazaki \& Fujita 2016). Additional data from the Framingham Offspring Study indicate that high levels of plasma aldosterone, albeit in the normal range, predict the development of hypertension (Garrison et al. 1987). In addition to hypertension, aldosterone is thought to mediate various other processes that contribute to cardiorenal disease, such as cardiac fibrosis, proteinuria and endothelial dysfunction, through its interaction with and activation of MR (Calhoun \& Sharma 2010). On the other hand, the glucocorticoid cortisol, perhaps acting through the MR, has also been proposed to contribute to obesity-associated hypertension (Varughese et al. 2014). Indeed, excess cortisol in Cushing's syndrome is associated with symptoms of metabolic syndrome, including abdominal obesity and hypertension. In many tissues, cortisol, which has the same affinity for the MR as aldosterone, is prevented from activating MR by the action of $11 \beta$-hydroxysteroid dehydrogenase-2 (11ßHSD2), which converts cortisol to its inactive metabolite, cortisone. Cortisone, in turn, can be reactivated by the enzyme 11ß-hydroxysteroid dehydrogenase-1 (11ßHSD1), and it has been proposed that excess cortisol, either in the serum or produced locally by dysregulation of $11 \beta \mathrm{HSD} 1 \mathrm{and} /$ or $11 \beta \mathrm{HSD} 2$, may be able to activate MR to contribute to obesity-associated hypertension (Varughese et al. 2014). Thus, multiple mechanisms may operate to mediate the hypertension linked to obesity. These additional mediators may explain the fact that not all studies have shown a correlation between adipose depots and aldosterone levels (e.g. O'Seaghdha et al. 2012) and/or between BMI and the response to an MR antagonist (Chapman et al. 2007, for example). Additional factors that may play a role in the observed heterogeneity of the data resulting from human studies include the proportion of male to female subjects (if as in mice (Huby et al. 2016), aldosterone contributes to a greater extent to obesity-associated hypertension in females (Goodfriend et al. 1999)), the absolute BMI of the subjects (i.e., normal weight versus overweight versus obese) and the relative adipose tissue distribution (e.g., visceral versus subcutaneous fat), as well as perhaps other parameters that have not yet been determined. Nevertheless, the results presented by Huby and coworkers (Huby et al. 2015), demonstrating that adipocyte-derived leptin likely mediates, at least in part, the effect of obesity on blood pressure by modulating aldosterone secretion, indicate that further studies are warranted.

\section{Declaration of interest}

The authors declare that there is no conflict of interest that could be perceived as prejudicing the impartiality of this review.

\section{Funding}

This work did not receive any specific grant from any funding agency in the public, commercial, or not-for-profit sector.
(C) 2016 Society for Endocrinology Printed in Great Britain
Published by Bioscientifica Ltd 


\section{Acknowledgements}

Dr Bollag is supported by a VA Research Career Scientist Award. The contents of this article do not represent the views of the Department of Veterans Affairs or the United States Government.

\section{References}

Briet M \& Schiffrin EL 2011 The role of aldosterone in the metabolic syndrome. Current Hypertension Reports 13 163-172. (doi:10.1007/ s11906-011-0182-2)

Calhoun DA \& Sharma K 2010 The role of aldosteronism in causing obesity-related cardiovascular risk. Cardiology Clinics 28 517-527. (doi:10.1016/j.ccl.2010.04.001)

Canale MP, Manca di Villahermosa S, Martino G, Rovella V, Noce A, De Lorenzo A \& Di Daniele N 2013 Obesity-related metabolic syndrome: mechanisms of sympathetic overactivity. International Journal of Endocrinology 2013 865965. (doi:10.1155/2013/865965)

Capponi AM 2002 Regulation of cholesterol supply for mineralocorticoid biosynthesis. Trends in Endocrinology \& Metabolism 13 118-121. (doi:10.1016/S1043-2760(01)00538-0)

Chapman N, Dobson J, Wilson S, Dahlof B, Sever PS, Wedel H, Poulter NR \& Anglo-Scandinavian cardiac outcomes trial I 2007 effect of spironolactone on blood pressure in subjects with resistant hypertension. Hypertension 49 839-845. (doi:10.1161/01. HYP.0000259805.18468.8c)

Ehrhart-Bornstein M, Lamounier-Zepter V, Schraven A, Langenbach J, Willenberg HS, Barthel A, Hauner H, McCann SM, Scherbaum WA \& Bornstein SR 2003 Human adipocytes secrete mineralocorticoidreleasing factors. PNAS 100 14211-14216. (doi:10.1073/ pnas.2336140100)

Engeli S \& Sharma AM 2000 Role of adipose tissue for cardiovascularrenal regulation in health and disease. Hormone and Metabolic Research 32 485-499. (doi:10.1055/s-2007-978675)

Garrison RJ, Kannel WB, Stokes J 3rd \& Castelli WP 1987 Incidence and precursors of hypertension in young adults: the Framingham Offspring Study. Preventive Medicine 16 235-251. (doi:10.1016/00917435(87)90087-9)

Goodfriend TL, Kelley DE, Goodpaster BH \& Winters SJ 1999 Visceral obesity and insulin resistance are associated with plasma aldosterone levels in women. Obesity Research 7 355-362. (doi:10.1002/j.1550-8528.1999.tb00418.x)

Huby AC, Antonova G, Groenendyk J, Gomez-Sanchez CE, Bollag WB, Filosa JA \& Belin de Chantemele EJ 2015 Adipocyte-derived hormone leptin is a direct regulator of aldosterone secretion, which promotes endothelial dysfunction and cardiac fibrosis. Circulation 132 2134-2145. (doi:10.1161/CIRCULATIONAHA.115.018226)

Huby AC, Otvos L Jr \& Belin de Chantemele EJ 2016 Leptin induces hypertension and endothelial dysfunction via aldosterone-dependent mechanisms in obese female mice. Hypertension 67 1020-1028. (doi:10.1161/HYPERTENSIONAHA.115.06642)
Kawarazaki W \& Fujita T 2016 The role of aldosterone in obesityrelated hypertension. American Journal of Hypertension 29 415-423. (doi:10.1093/ajh/hpw003)

Malendowicz LK, Nussdorfer GG \& Markowska A 1997 Effects of recombinant murine leptin on steroid secretion of dispersed rat adrenocortical cells. Journal of Steroid Biochemistry and Molecular Biology 63 123-125. (doi:10.1016/S0960-0760(97)00082-4)

Norata GD, Pirillo A, Callegari E, Hamsten A, Catapano AL \& Eriksson P 2003 Gene expression and intracellular pathways involved in endothelial dysfunction induced by VLDL and oxidised VLDL. Cardiovascular Research 59 169-180. (doi:10.1016/S0008-6363(03)00335-3)

O'Seaghdha CM, Hwang SJ, Vasan RS, Larson MG, Hoffmann U, Wang TJ \& Fox CS 2012 Correlation of renin angiotensin and aldosterone system activity with subcutaneous and visceral adiposity: the framingham heart study. BMC Endocrine Disorders 123. (doi:10.1186/1472-6823-12-3)

Rondinone CM 2006 Adipocyte-derived hormones, cytokines, and mediators. Endocrine 29 81-90. (doi:10.1385/endo:29:1:81)

Saha S, Bornstein SR, Graessler J \& Kopprasch S 2012a Very-low-density lipoprotein mediates transcriptional regulation of aldosterone synthase in human adrenocortical cells through multiple signaling pathways. Cell and Tissue Research 348 71-80. (doi:10.1007/s00441012-1346-3)

Saha S, Willenberg HS, Bornstein SR, Graessler J \& Kopprasch S $2012 b$ Diabetic lipoproteins and adrenal aldosterone synthesis - a possible pathophysiological link? Hormone and Metabolic Research 44 239-244. (doi:10.1055/s-0031-1295459)

Saha S, Schwarz PE, Bergmann S, Bornstein SR, Graessler J \& Kopprasch S 2013 Circulating very-low-density lipoprotein from subjects with impaired glucose tolerance accelerates adrenocortical cortisol and aldosterone synthesis. Hormone and Metabolic Research 45 169-172. (doi:10.1055/s-0032-1323844)

Salzmann C, Otis M, Long H, Roberge C, Gallo-Payet N \& Walker CD 2004 Inhibition of steroidogenic response to adrenocorticotropin by leptin: implications for the adrenal response to maternal separation in neonatal rats. Endocrinology 145 1810-1822. (doi:10.1210/en.20031514)

Tsai YY, Rainey WE, Pan ZQ, Frohman MA, Choudhary V \& Bollag WB 2014 Phospholipase D activity underlies very-low-density lipoprotein (VLDL)-induced aldosterone production in adrenal glomerulosa cells. Endocrinology 155 3550-3560. (doi:10.1210/en.2014-1159)

Varughese AG, Nimkevych O \& Uwaifo GI 2014 Hypercortisolism in obesity-associated hypertension. Current Hypertension Reports 16443. (doi:10.1007/s11906-014-0443-y)

Xing Y, Cohen A, Rothblat G, Sankaranarayanan S, Weibel G, Royer L, Francone OL \& Rainey WE 2011 Aldosterone production in human adrenocortical cells is stimulated by high-density lipoprotein 2 (HDL2) through increased expression of aldosterone synthase (CYP11B2). Endocrinology 152 751-763. (doi:10.1210/en.2010-1049)

Xing Y, Rainey WE, Apolzan JW, Francone OL, Harris RB \& Bollag WB 2012 Adrenal cell aldosterone production is stimulated by very-lowdensity lipoprotein (VLDL). Endocrinology 153 721-731. (doi:10.1210/ en.2011-1752)

Received in final form 25 May 2016

Accepted 31 May 2016

Accepted Preprint published online 1 June 2016 http://joe.endocrinology-journals.org

DOI: 10.1530/JOE-16-0160
๑) 2016 Society for Endocrinology Printed in Great Britain 